ORIGINAL ARTICLE

\title{
Exercise following a sport induced concussion
}

\author{
B Gall, W S Parkhouse, D Goodman
}

Br J Sports Med 2004;38:773-777. doi: 10.1136/bjsm.2003.009530

See end of article for authors' affiliations

.....................

Correspondence to:

Dr W S Parkhouse, Simon

Fraser University, School

of Kinesiology, 8888

University Blvd, Burnaby,

BC V5A 1S6, Canada;

parkhouse@sfu.ca

Accepted

25 November 2003

\begin{abstract}
Objective: To determine if an athlete's capacity to perform exercise is impaired following concussion and whether this would be reflected by an altered heart rate response.

Methods: Of the 14 concussed athletes, nine missed playing time as a direct result of their concussion and five did not. The concussed athletes performed an exercise protocol on a cycle ergometer within 72 hours of being asymptomatic at rest and a second test at 5 days following the previous assessment. Matched controls $(n=14)$ were tested using the same time line. The exercise protocol consisted of a 2 minute warm up, 10 minute, low-moderate intensity, steady state exercise session, and a high intensity interval protocol. The interval protocol consisted of a 40 second high intensity bout, followed by a 40 second rest period. This protocol continued until the participant had reached volitional fatigue.

Results: The number of exercise bouts completed was not significantly different from their matched controls. However, concussed athletes who missed playing time had a significantly higher heart rate during the steady state exercise session. During this same period, they also exhibited a greater rise in heart rate over time.

Conclusion: These findings indicate that exercise capacity is unaffected in concussed athletes who are asymptomatic at rest. However, their heart rate response to submaximal exercise is increased.
\end{abstract}

A lthough concussion is recognised as one of the most frequently occurring injuries in contact sport, ${ }^{1}$ it is difficult for health practitioners to determine when an athlete can safely return to play. This difficulty primarily arises from the lack of clear, sensitive, and objective prognostic markers. ${ }^{2}$ More often than not, health practitioners must rely on the self reported symptoms from the athlete and good clinical judgement as their primary tool for determining when an athlete should return to play. ${ }^{3}$ Unfortunately, relying on these symptoms is problematic, particularly in sport, and returning an athlete too soon may place them at risk for a further neurological injury, ${ }^{45}$ which could result in long term neurological sequelae. ${ }^{6-9}$

A concussed athlete's response to physiological stress may provide valuable insight into determining when they have recovered from concussion. However, few studies have investigated this relationship between mild head injury and physiological stress. ${ }^{10}$ In one study, concussed athletes demonstrated an increase in heart rate when exposed to mental stress, potentially indicating an autonomic dysfunction. ${ }^{11}$ In addition, neuroautonomic cardiovascular dysfunction has been well documented in severe head trauma. ${ }^{12}{ }^{13}$ Thus the purpose of this study was to assess the ability of athletes to perform exercise and their physiological response to that exercise, following an period of being asymptomatic at rest after a sport induced concussion.

\section{METHODS \\ Subjects}

A total 147 junior hockey players was given a written and oral briefing of the study and the risks involved prior to signing the informed consent. The study was approved by the university research ethics review committee. Baseline data collected included a medical questionnaire, concussion history, and a signs and symptoms questionnaire similar to that of Lovell and Collins. ${ }^{14}$

Fourteen of the 147 participants had sustained a concussion. Their corresponding matched controls $(n=14)$ were team mates chosen to match, as closely as possible, the concussed athletes (table 1). Team trainers in collaboration with game observers used the concussion symptom guidelines of the Canadian Hockey Association ${ }^{15}$ to determine whether or not an athlete had sustained a concussion.

The concussed athletes performed the exercise protocol within 72 hours of being asymptomatic at rest. The test was repeated 5 days after the initial exercise assessment.

\section{Symptoms}

The same signs and symptoms questionnaire administered at baseline was given to the concussed athletes at $1.8(0.2)$ days after injury. If the athlete was still symptomatic in relation to their baseline questionnaire, follow ups were conducted at 3 day intervals until the athlete was asymptomatic at rest or had returned to play. Once cleared to participate in exercise testing, the questionnaire was filled out prior to and 5 minutes following exercise at each testing session.

The questionnaire consisted of 20 signs and symptoms associated with concussion that are graded on a scale of 0-6 by the athlete. ${ }^{14}$ For the purpose of analysis between baseline and the symptoms reported at 2 days post-injury, a composite score was constructed using five symptoms that had been previously validated as indicators of concussion: ${ }^{3}{ }^{16-18}$ headache, difficulty concentrating, difficulty remembering, nausea, and dizziness. Because the signs and symptoms questionnaire was administered 5 minutes following exercise, a physical composite score comprised of only nausea, dizziness, and headache was used in the analysis comparing before and after exercise.

\section{Exercise protocol}

All 28 athletes were required to fill out a modified PAR-Q before each testing session to ensure their health status had not changed from the start of the season. The exercise protocol was performed on a constant load cycle ergometer (Jaeger ER800, Germany). The participants began the protocol with a 2 minute warm up, pedalling at a frequency of 50-60 rpm with a load of $40 \mathrm{~W}$. Following the warm up,

Abbreviations: HR, heart rate; MT, missed time; NMT, no missed time 
Table 1 Subject characteristics

\begin{tabular}{|c|c|c|c|c|}
\hline & Age (years) & Height $(\mathrm{cm})$ & Weight (kg) & $\begin{array}{l}\text { No. of prior } \\
\text { concussions* }\end{array}$ \\
\hline \multicolumn{5}{|l|}{ Missed time } \\
\hline Concussed & $17.8(0.5)$ & $179.4(1.4)$ & $80.2(4.1)$ & $2.0(0.5)$ \\
\hline Matched controls & $18.7(0.4)$ & $181.5(1.6)$ & 76.5 (1.8) & $1.3(0.5)$ \\
\hline \multicolumn{5}{|l|}{ No missed time } \\
\hline Concussed & $18.8(0.8)$ & $181.3(2.7)$ & $79.6(2.2$ & $1.8(0.6)$ \\
\hline Matched controls & $19.0(0.8)$ & $179.7(2.5)$ & $81.0(3.2)$ & $0.8(0.6)$ \\
\hline
\end{tabular}

the athletes engaged in a 10 minute, low-moderate intensity, steady state exercise bout (80-90 rpm against a constant load of $1.5 \mathrm{~W} / \mathrm{kg}$ of body weight). This was immediately followed by a high intensity interval session consisting of repeated 40 second high intensity bouts at $4.7 \mathrm{~W} / \mathrm{kg}$ with a pedalling frequency of 90-100 rpm, followed by a 20 second free pedal $(30 \mathrm{~W})$ and a subsequent 20 second rest period. The high intensity exercise bouts were repeated until the athlete could no longer maintain the workload. Recovery consisted of a 1 minute free pedal with a load of $30 \mathrm{~W}$, followed by a resting state for an additional 4 minutes. The number of high intensity exercise bouts completed was recorded. This exercise protocol was designed to reflect the high intensity interval activity observed in the sport of ice hockey.

\section{Heart rate and blood lactate measures}

A three lead CM5 configuration was used to record ECG data. The ECG signal was sampled at a frequency of $976 \mathrm{~Hz}$ through a Burdick EK-10 Electrocardiograph and simultaneously stored on a laptop using the WINDAQ ${ }^{\circledR}$ data acquisition system. ECG data was collected throughout the entire exercise protocol in addition to a 5 minute recovery sample immediately following the completion of the high intensity exercise protocol. To ensure that heart rate (HR) was at steady state, only minutes 2-9 from the lowmoderate intensity steady state exercise bout were used for analysis. Five minute resting samples were also taken prior to exercise and at $30 \mathrm{~min}$ post-exercise. Blood was extracted from the fingertip prior to exercise and at 1 and 3 minutes post-exercise and analysed for lactate concentration (Accutrend ${ }^{\circledast}$ Lactate analysis system).

\section{Data analysis}

The concussed athletes and their corresponding matched controls were divided into two cohorts as outlined in table 1 ; those who missed playing time $(n=9)$ as a direct result of their concussion (MT cohort) and those $(n=5)$ who did not (NMT cohort). Using the composite score, baseline symptoms of the concussed athletes was compared with their reported symptoms at 2 days post-injury, using a 2 (missed time) $\times$ 2 (time)-2 factor mixed model one way analysis of variance. The number of exercise bouts completed was analysed using a 2 (concussion) $\times 2$ (missed time) $\times 2$ (test) -3 factor mixed model one way analysis of variance, with repeated measures on the test factor. In addition, four way mixed model, one way analyses of variance with repeated measures on the test and exercise/time factors were used for the remaining variables. When a significant $\mathrm{F}$ value was detected for main effects, a Bonferroni corrected pairwise comparison among estimated marginal means was used to determine where differences existed. Significance was set at a level of $\mathrm{p}<0.05$ and all data are presented as means (SE).

\section{RESULTS}

\section{Subjects}

All of the 14 concussed athletes were deemed to have sustained a concussion based on the reports filed by the team trainers and game observers. Six of the concussed athletes from the MT cohort were unable to participate in the exercise protocol at $1.7(0.2)$ days post-injury because their symptoms had not yet returned to baseline. Of the nine concussed athletes in this group, four experienced loss of consciousness, while four others exhibited post-traumatic amnesia immediately following their injury. The remaining athlete from this group reported no loss of consciousness, but had missed playing time as a direct result of his injury. In contrast, the symptoms from all concussed athletes of the NMT cohort had returned to baseline levels 2.0 (0.5) days after the injury. In addition, only one of the concussed athletes in the NMT group reported post-traumatic amnesia and none experienced loss of consciousness. An average of 6.7 (1.8) days for the MT cohort and 2.0 (0.5) days for the NMT group had elapsed from the time of injury to the first exercise test.

\section{Exercise bouts and blood lactate levels}

The number of high intensity exercise bouts completed by the concussed athletes was not significantly different from their matched controls in either cohort (table 2); however, a significant test by concussion interaction was detected. Specifically, the concussed group completed 8.0 (1.1) bouts at the first exercise test, and 6.8 (1.1) bouts at test 2 . In contrast, their matched controls demonstrated the opposite response, completing $7.6(0.7)$ bouts at test 1 , and $8.2(0.9)$ bouts at test 2 . The concussed participants and the matched controls of the NMT group were able to perform an average of 9.4 (1.1) exercise bouts across both exercise tests, before reaching volitional fatigue. This was significantly higher than the $6.6(0.7)$ bouts completed by the MT cohort.

In response to the high intensity exercise protocol, blood lactate levels rose significantly from an average resting value of $1.7(0.1) \mathrm{mmol} / \mathrm{l}$ to $11.4(0.4) \mathrm{mmol} / \mathrm{l}$ immediately after exercise. No significant difference in blood lactate levels at either rest or post-exercise was observed between the concussed group and their matched controls (table 2).

\section{Symptoms}

At baseline, both the MT and NMT concussed athletes had similar composite scores of 3.3 (1.4) and 3.6 (2.7) respectively. Following their reported concussion, the MT athletes had a score of 6.8 (2.2), whereas the NMT athletes had a composite score of $3.6(2.0)$. Neither the main effect $(3.6$ (1.9) NMT; 5.0 (1.5) MT) or interactions were statistically significant.

At the first exercise test, the MT concussed athletes and their matched controls respectively reported a physical symptoms composite score of $1.9(0.7)$ and $1.0(0.5)$ at rest (fig 1). Following exercise at the same testing session, the 
Table 2 The number of exercise bouts completed and blood lactate levels $(\mathrm{mmol} / \mathrm{l})$ at rest and post-exercise during both testing sessions

\begin{tabular}{|c|c|c|c|c|c|c|}
\hline & \multicolumn{3}{|l|}{ Test 1} & \multicolumn{3}{|l|}{ Test 2} \\
\hline & $\begin{array}{l}\text { Exercise } \\
\text { bouts }\end{array}$ & $\begin{array}{l}\text { Resting } \\
\text { lactate }\end{array}$ & $\begin{array}{l}\text { Post- } \\
\text { exercise } \\
\text { lactate }\end{array}$ & $\begin{array}{l}\text { Exercise } \\
\text { boutst }\end{array}$ & $\begin{array}{l}\text { Resting } \\
\text { lactate }\end{array}$ & $\begin{array}{l}\text { Post- } \\
\text { exercise } \\
\text { lactate }^{*}\end{array}$ \\
\hline \multicolumn{7}{|l|}{ Missed time } \\
\hline Concussed $(n=8)$ & $6.6(1.1)$ & $2.2(0.4)$ & $10.9(0.8)$ & $5.3(0.9)$ & $1.7(0.2)$ & $12.5(0.6)$ \\
\hline Matched controls ( $n=8$ ) & $7.4(0.9)$ & $1.6(0.3)$ & $11.1(0.7)$ & $7.3(1.2)$ & $1.8(0.2)$ & $11.1(0.9)$ \\
\hline \multicolumn{7}{|l|}{ No missed time } \\
\hline Concussed $(n=5)$ & $10.2(2.2)$ & $1.5(0.1)$ & $12.2(1.2)$ & $9.2(2.0)$ & $1.6(0.3)$ & $11.8(1.2)$ \\
\hline Matched controls $(n=4)$ & $8.0(1.1)$ & $1.4(0.2)$ & $10.0(2.2)$ & $10.0(0.9)$ & $1.5(0.2)$ & $12.9(1.1)$ \\
\hline
\end{tabular}

Values are means (SE). ${ }^{*} p<0.01$ significant exercise effect across both tests; $t p<0.05$ missed time main effect across both tests.

concussed athletes and their controls demonstrated a nonsignificant increase in symptom severity. The MT concussed athletes reported a score of 4.3 (1.8), whereas the MT controls reported less severe symptoms with a score of 2.3 (0.9). Similar results were also seen 5 days later (MT concussed 3.0 (1.1); MT control 2.5 (1.0)). No difference was found between the MT and NMT cohorts or between the concussed and control athletes of the NMT group (fig 2).

\section{Heart rate}

The maximum heart rate (MHR) achieved by the MT concussed athletes was higher, but not statistically different, at both testing sessions relative to their matched controls (table 3). Across both tests a significant rise in heart rate was observed during the course of the steady state exercise session (figs 3,4). The rise in HR in the MT group over both exercise tests was found to be significantly greater than their matched controls (fig 3). The MT concussed athletes also had a significantly higher average heart rate than their matched controls over the 8 minute time period across both tests (126 (3.4) beats/min MT concussed; 116.0 (1.9) beats/min for MT control). This significant difference was not apparent between the concussed and control groups of the NMT cohort (fig 4).

HR dropped significantly during the 5 minutes immediately following exercise cessation (figs 5, 6). However, the average HR of the concussed athletes during this 5 minute time frame was not significantly different from their matched controls in either the MT or NMT cohort. Similarly no concussion by time interaction effect was detected, indicating that the decrease in HR over the 5 minute recovery period was similar in both groups (figs 5, 6).

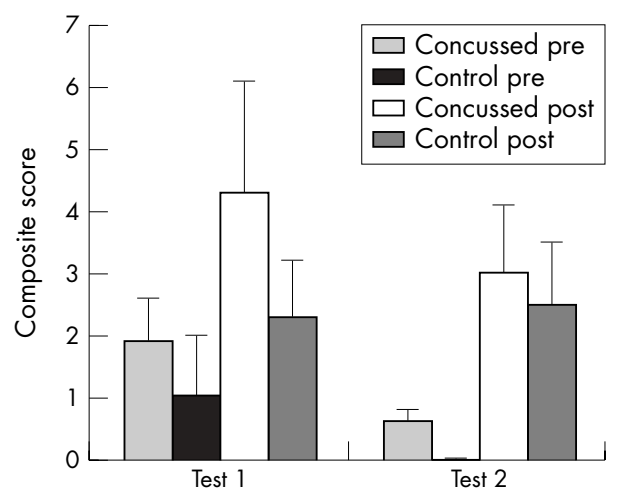

Figure 1 Composite score of reported symptoms before and after exercise at both follow up tests for the MT cohort. Concussed, $n=9$; matched control, $n=8$.

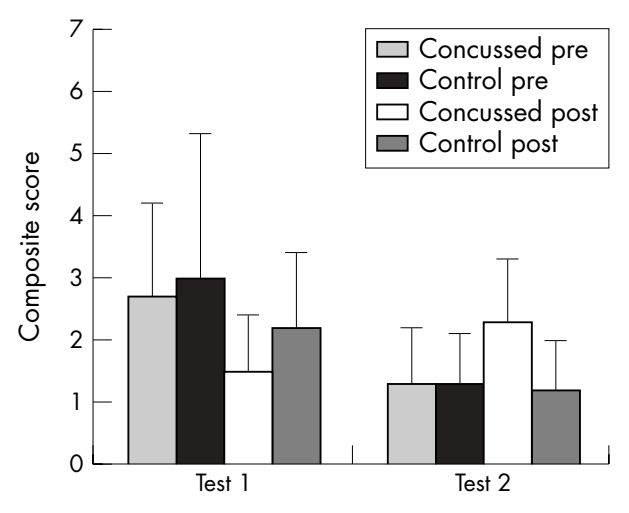

Figure 2 Composite score of reported symptoms before and after exercise at both follow up tests for the NMT cohort. Concussed, $n=4$; matched control, $n=5$.

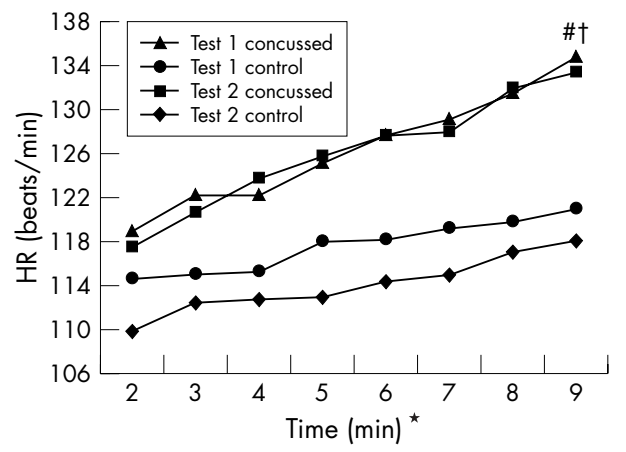

Figure 3 Heart rate for the MT cohort, from minute 2 to minute 9 during the 10 minute, low-moderate, steady state exercise period; $n=7$ for concussed athletes and matched controls. *Significant time main effect $(p<0.05)$; \#significant time by concussion interaction effect $(p<0.05)$; tsignificant concussion main effect $(p<0.05)$.

\section{DISCUSSION}

The number of exercise bouts completed by the concussed athletes was not significantly different from their matched controls. However, concussed athletes who missed playing time had a significantly higher heart rate during the lowmoderate, steady state exercise period, and they also exhibited a greater rise in heart rate over time. Despite the difference in cardiovascular response to exercise, concussed athletes and their matched controls reported similar symptoms before and after exercise. Contrary to our hypothesis, the capacity to perform high intensity interval activity was unaltered by concussion. The effort put forth by the concussed and control athletes at both testing sessions was 


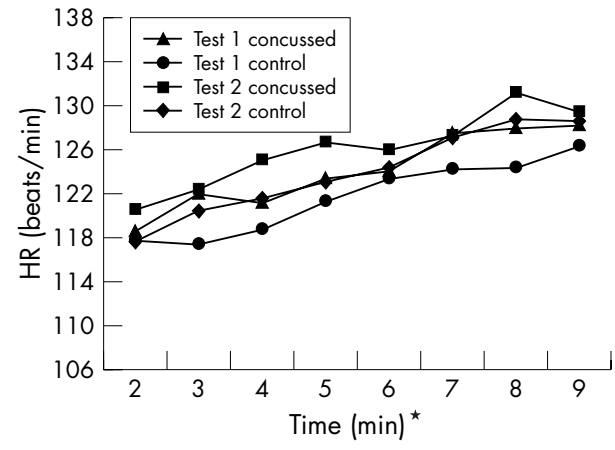

Figure 4 Heart rate for the NMT cohort, from minute 2 to minute 9 during the 10 minute, low-moderate, steady state exercise period. Concussed, $n=5$; matched control, $n=3$. *Significant time main effect $(p<0.05)$.

comparable and near maximum intensity based on the number of exercise bouts completed, blood lactate levels post-exercise, and maxiumum HR achieved.

It is possible that the exercise capacity test used in this study may have been too gross a measure to detect a difference between the concussed athletes and their matched controls after a period of being asymptomatic at rest. An additional factor influencing the exercise capacity of the concussed athletes might be the elapsed time between the time of the injury and the first exercise test. Specifically, the concussed athletes of the MT cohort conducted their first exercise test at slightly less than 1 week post-injury and within $72 \mathrm{hrs}$ of being asymptomatic at rest. This may have been too late in the recovery phase for the effects of concussion to influence exercise capacity.

The significant difference in HR during steady state exercise is of considerable interest. The use of symptoms to progress through a stepwise exercise programme is strongly recommended by the Concussion in Sports Group when managing a concussed athlete's return to play. ${ }^{4}$ However, no detectable difference was found in the reported symptoms of our concussed athletes, despite these individuals demonstrating an adverse cardiovascular response to exercise. Consequently, while it appears that the concussed athlete has not yet fully recovered from their injury, this is not reliably detected by the reported symptoms.

It may be argued that a detraining effect, rather than concussion, is responsible for the elevated HR. However previous studies suggest that an average elapsed time (from injury to the first exercise test) of less than l week is insufficient to induce a change in HR of this magnitude. ${ }^{19-21}$ The current study shows an $8 \%$ increase in submaximal HR in less than 1 week. This increase in HR is consistent with

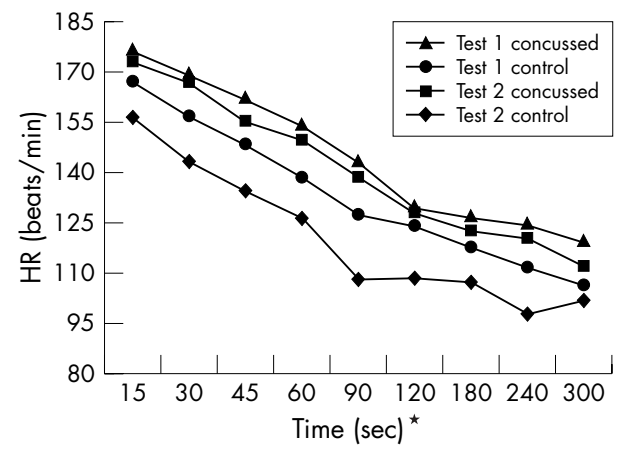

Figure 5 Heart rate for the MT cohort during the 5 minute recovery stage immediately following the cessation of the high intensity interval protocol. Concussed, $n=7$; matched control, $n=4$. * Significant time main effect $(p<0.05)$.

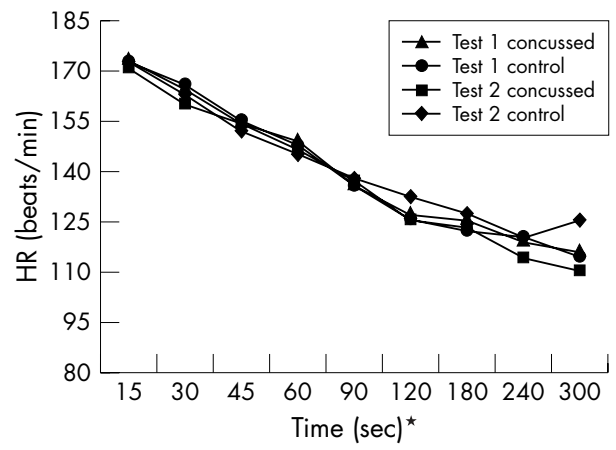

Figure 6 Heart rate for the NMT cohort, during the 5 minute recovery stage immediately following the cessation of the high intensity interval protocol. Concussed, $n=5$; matched control, $n=3$. *Significant time main effect $(p<0.05)$.

previous literature demonstrating abnormality in cardiovascular functioning following severe head trauma. ${ }^{1-13}$

It has been stated that athletes recovering from a sport induced concussion can probably return to play 5-7 days post-injury provided their symptoms have resolved. ${ }^{22}$ However, the speed of symptom resolution is dependent upon the individual. ${ }^{22}$ In the present study, HR was still elevated at the second exercise test 12 days post-injury in the concussed athletes who missed playing time. If indeed this elevated heart rate reflects cardiovascular autonomic dysfunction, a longer time frame is required to monitor these athletes to full recovery.

Of particular interest is a study by Goldstein et $a l^{13}$ the results of which suggest that the degree of uncoupling

Table 3 Heart rate (beats/min) at rest and post-exercise, and maximum heart rate achieved during high intensity exercise, at both testing sessions

\begin{tabular}{|c|c|c|c|c|c|c|}
\hline & \multicolumn{3}{|l|}{ Test 1} & \multicolumn{3}{|l|}{ Test 2} \\
\hline & RHR & MHR & PEHR & RHR & MHR $^{*}$ & PEHR $\dagger$ \\
\hline \multicolumn{7}{|l|}{ Missed time } \\
\hline Concussed $(n=9)$ & $68.2(3.7)$ & $183.4(1.8)$ & $81.0(2.9)$ & $65.2(3.1)$ & 182.4 (1.9) & $80.4(3.0)$ \\
\hline $\begin{array}{l}\text { Matched control }(n=7) \\
\text { No missed time }\end{array}$ & $63.2(2.4)$ & $173.6(2.4)$ & $75.8(2.5)$ & $64.4(3.1)$ & $173.5(3.3)$ & $74.5(3.1)$ \\
\hline Concussed $(n=4)$ & $62.0(3.1)$ & $180.0(9.8)$ & $78.9(6.1)$ & $61.2(2.8)$ & $179.0(10.4)$ & $78.2(7.5)$ \\
\hline Matched control $(n=4)$ & $67.9(2.1)$ & $182.6(6.7)$ & 73.7 (2.7) & $66.6(6.9)$ & $188.1(2.3)$ & $79.4(3.1)$ \\
\hline
\end{tabular}

RHR, resting heart rate; PEHR, post-exercise heart rate; MHR, maximum heart rate. Values are reported as means (SE). ${ }^{*} p<0.01$ significantly different from both RHR and PEHR across both tests; $\uparrow p<0.01$ significantly different from RHR across both tests. 


\section{WHAT IS ALREADY KNOWN ON THIS TOPIC}

- Concussed athletes demonstrated an increase in heart rate when exposed to mental stress, potentially indicating a neuroautonomic dysfunction, which is well documented in severe head trauma.

- Study of concussion symptoms and responses to physical exercise is recommended in determining whether an athlete should return to play following a concussion.

\section{WHAT THIS STUDY ADDS}

- This is the first study to identify potential cardiovascular neuroautonomic control abnormalities during exercise in concussed athletes following period of being asymptomatic at rest.

- It also demonstrates that these athletes' ability to perform maximum exercise is unaffected following period of being asymptomatic at rest after concussion.

between the autonomic and cardiovascular systems is proportional to the severity of the neurological injury. In contrast to the concussed athletes of the MT cohort, those from the NMT group did not demonstrate a difference in steady state heart rate in relation to their controls. Based on Goldstein's findings, it could be suggested that the concussions sustained in the NMT group were not as severe. This assumption is further supported by the fact that the symptoms of this group had returned to baseline values at approximately 2 days post-injury. It is possible that, unlike traumatic brain injury, the severity of the neurological damage associated with concussion may be insufficient to induce cardiovascular changes at rest. However, under conditions of physiological stress, such as exercise, cardiovascular abnormalities may become more explicit.

In contrast to the anecdotal evidence, the concussed athletes from the present study demonstrated no difference in symptoms before and after exercise. It is unknown as to whether or not the lack of symptom aggravation is due to misrepresentation of symptoms from the athlete as a result of "contra-malingering", or if indeed they were not perturbed as a function of exercise. Thus the use of symptoms, particularly following exercise, requires further research.

In summary, exercise capacity is unaffected in concussed athletes following a period of being asymptomatic at rest. However, the abnormal cardiovascular response to submaximal exercise appears to indicate impairment in neuroautonomic cardiovascular regulation. Furthermore, the degree of cardiovascular abnormality appears to be representative of concussion severity. Consequently, it is anticipated that this research will stimulate additional exploration into the relationship between neuroatuonomic cardiovascular functioning following a concussion.

\section{ACKNOWLEDGEMENTS}

The authors would like to thank Harold Brittain, President, Pacific International Junior B Hockey League (PIJHL), owners, coaches, trainers, staff and players for their participation in this study; C Matsushita, C Alkins, T Swanson, L Cunnigham, C Brumpton and J Litt for their assistance in data collection. We also wish to thank Finning International, Canon Imaging Solutions and Northern Lite Manufacturing Ltd. for their contribution to this research. Funding for this project was provided by the Canadian Institutes of Health Research (CIHR)

\section{Authors' affiliations}

B Gall, W S Parkhouse, D Goodman, Simon Fraser University, Burnaby, Canada

Conflict of interest: none declared

\section{REFERENCES}

1 Cantu RC. Head injuries in sport. Br J Sports Med 1996;30:289-96.

2 Johnston KM, McCrory P, Mohtadi NG, et al. Evidence-based review of sportrelated concussion: Clinical science. Clin J Sport Med 2001;11:150-9.

3 McCrory PR, Johnston KM. Acute clinical symptoms of concussion. Physician Sports Med 2002;30:43-7.

4 Aubry M, Cantu R, Dovrak J, et al. Summary and agreement statement of the first International Conference on Concussion in Sport, Vienna 2001. Br J Sports Med 2002;36:6-10.

5 Baker RJ, Patel DR. Sports related mild traumatic brain injury in adolescents. Indian J Pediatr 2000;67:317-21

6 Rabadi MH, Jordan BD. The cumulative effect of repetitive concussion in sports. Clin J Sports Med 2001;11:194-8.

7 Gaetz M, Goodman D, Weinberg H. Electrophysiological evidence for the cumulative effects of concussion. Brain Inj 2000;14:1077-88.

8 Gaetz M, Weinberg $\mathrm{H}$. Electrophysiological indices of persistent postconcussion symptoms. Brain Inj 2000;14:815-32.

9 Gronwall D, Wrightson P. Cumulative effect of concussion. Lancet 1975;2:995-7.

10 Ewing R, McCarthy D, Gronwall D, et al. Persisting effects of minor head injury observable during hypoxic stress. J Clin Neuropsychol 1980;2:147-55.

11 Hanna-Pladdy B, Berry ZM, Bennett T, et al. Stress as a diagnostic challenge for postconcussive symptoms: Sequelae of mild traumatic brain injury or physiological stress response. Clin Neuropsychologist 2001;15:289-304.

12 Clifton GL, Robertson CS, Kyper K, et al. Cardiovascular response to severe head trauma. J Neurosurg 1983;59:447-54.

13 Goldstein B, Toweill D, Susanna L, et al. Uncoupling of the autonomic and cardiovascular systems in acute brain injury. Am J Physiol 1998:275:R1 287-92.

14 Lovell M, Collins MW. Neuropsychological assessment of the college football player. J Head Trauma Rehabil 1998;13:9-26.

15 Canadian Hockey Association. Gatorade Canadian Hockey Safety Program. Ottawa: Canadian Hockey Association, 1999.

16 Erlanger D, Kaushik T, Cantu R, et al. Symptom-based assessment of severity of a concussion. J Neurosurg 2003;98:477-84.

17 Maddocks DL, Dicker GD, Saling MM. The assessment of orientation following concussion in athletes. Clin J Sport Med 1995:5:32-5.

18 McCrory PR, Ariens M, Berkovic SF. The nature and duration of acute concussive symptoms in Australian football. Clin J Sport Med 2000;10:235-8

19 Fardy P. Effects of soccer training and detraining upon selected cardiac and metabolic measures. Res Q Exerc Sport 1969;40:502-8.

20 Mujika I, Padilla S. Cardiorespiratory and metabolic characteristics of detraining in humans. Med Sci Sports Exerc 2000;33:413-21.

21 Penny G, Wells M. Heart rate, blood pressure, serum lactate, and serum cholesterol changes after the cessation of training. J Sports Med 1975; 15:223-8.

22 Grindel SH, Lovell MR, Collins MW. The assessment of sport-related concussion: The evidence behind neuropsychological testing and management. Clin J Sport Med 2001;11:134-43. 